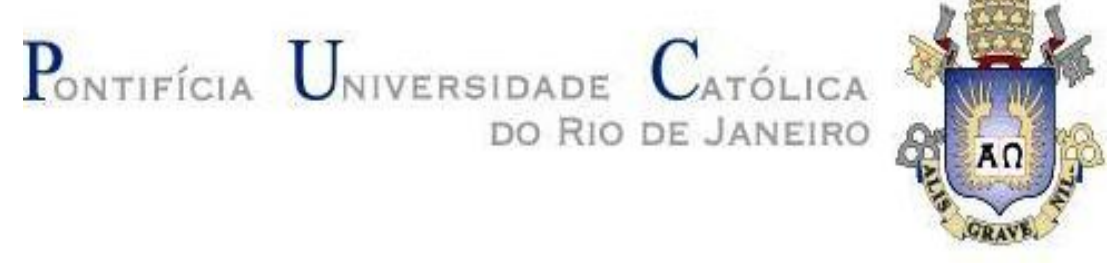

Meryelem Tania Churampi Arellano

Tratamento de efluentes contendo cianeto por oxigênio singlete gerado através da reação de peróxido de hidrogênio e hipoclorito de sódio

Dissertação de Mestrado

Dissertação apresentada como requisito parcial para obtenção do título de Mestre pelo Programa de PósGraduação em Engenharia de Materiais e de Processos Químicos e Metalúrgicos do Departamento de Engenharia de Materiais da PUCRio.

Orientador: Prof. Luiz Alberto Cesar Teixeira 


\section{Tratamento de efluentes contendo cianeto por oxigênio singlete gerado através da reação de peróxido de hidrogênio e hipoclorito de sódio}

Dissertação apresentada como requisito parcial para obtenção do grau de Mestre pelo Programa de Pósgraduação em Engenharia de Materiais e de Processos Químicos e Metalúrgicos do Departamento de Engenharia de Materiais do Centro Técnico Científico da PUC-Rio. Aprovada pela Comissão Examinadora abaixo assinada.

Prof $^{\circ}$. Luiz Alberto Cesar Teixeira Orientador e Presidente Departamento de Engenharia de Materiais - PUC Rio

Profa Lídia Yokoyama Universidade Federal do Rio de Janeiro

Profa Fabiana Valéria da Fonseca Araujo Universidade Federal do Rio de Janeiro

Prof $^{\circ}$. José Eugenio Leal Coordenador Setorial de Pós-Graduação do Centro Técnico Científico da PUC- Rio 
Todos os direitos reservados. É proibida a reprodução total ou parcial do trabalho sem autorização da universidade, do autor e do orientador.

Meryelem Tania Churampi Arellano

Graduou-se em Engenharia Química pela Universidade Nacional do Centro do Perú em 2005.

Ficha Catalográfica

Arellano, Meryelem Tania Churampi

Tratamento de efluentes contendo cianeto por oxigênio singlete gerado através da reação de peróxido de hidrogênio e hipoclorito de sódio/ Meryelem Tania Churampi Arellano; orientador: Luiz Alberto Cesar Teixeira. - 2012.

141 f. : il. (color.) ; $30 \mathrm{~cm}$

Dissertação (mestrado)-Pontifícia Universidade Católica do Rio de Janeiro, Departamento de Engenharia de Materiais, 2012.

Inclui bibliografia

1. Engenharia de materiais - Teses. 2. Oxigênio singlete. 3. Oxidação do cianeto. 4. Peróxido de hidrogênio. I. Teixeira, Luiz Alberto Cesar. II. Pontifícia Universidade Católica do Rio de Janeiro. Departamento de Engenharia de Materiais. III. Título. 
Dedico meu trabalho a Deus, aos meus adoráveis pais Zoraida e Nery, a meus irmãos Luis, Miriam, Edith, Karito, e meus sobrinhos Jason e Marcelita pelo amor e apoio incondicional nos momentos de felicidade e dificuldade. 


\section{Agradecimentos}

Agradeço a Deus por toda a força nos momentos difíceis e pelas conquistas.

Especial agradecimento ao meu orientador, Professor Luiz Alberto Cesar Teixeira, por me acolher desde o primeiro momento que o conheci e confiar nas minhas capacidades, por me oferecer este maravilhoso projeto e a confiança para conseguir desenvolvê-lo. Agradeço a ele, também, por toda a orientação, pela paciência, carinho, incentivo, pelo valioso apoio acadêmico e moral para a realização deste trabalho. Obrigada por me incentivar, pela valiosa ajuda que me brindou no transcurso do mestrado e por ser uma pessoa agradável, atenciosa e sincera.

A meu colega Renzon Cosme Pecho, obrigada pelo grande apoiou, por me transmitir seus conhecimentos, pela ajuda valiosa no laboratório, por me ensinar a atuar, pensar e trabalhar de maneira individual, estando sempre presente, corrigindo o meus erros em todo momento durante o desenvolvimento deste projeto e por contribuir em muito no meu crescimento acadêmico.

Ao CAPES e a PUC-Rio, pelo apoio concedido para a realização deste trabalho.

À Peróxidos do Brasil Ltda pelo apoio a nosso laboratório e nossas pesquisas.

À Rosana, pela paciência, carinho, amizade e por toda sua ajuda no mestrado.

Aos Professores, funcionários e colegas do Departamento de Engenharia de Materiais.

Ao grupo de trabalho de pesquisa pelo suporte nas horas que precisei e por oferecer também seus conhecimentos. 


\section{Resumo}

Arellano, Meryelem Tania Churampi; Luiz Alberto Cesar Teixeira. Tratamento de efluentes contendo cianeto por oxigênio singlete gerado através da reação de peróxido de hidrogênio e hipoclorito de sódio. Rio de Janeiro, 2012. 141 p. Dissertação de Mestrado - Departamento de Engenharia de Materiais e Metalúrgica, Pontifícia Universidade Católica do Rio de Janeiro.

O presente trabalho teve como objetivo estudar a viabilidade da aplicação do processo oxidativo avançado que utiliza oxigênio singlete, gerado quimicamente por peróxido de hidrogênio e hipoclorito de sódio, para oxidar o cianeto livre. O processo foi estudado em batelada, simulando uma solução sintética de $\mathrm{KCN}$ com características de $\mathrm{pH}$ e concentração similares às condições típicas de um efluente real. A combinação aquosa do $\mathrm{H}_{2} \mathrm{O}_{2}$ e $\mathrm{NaClO}$ para gerar oxigênio singlete, foi eficaz para oxidar o cianeto, em uma faixa de pH 9 a 11. Com concentrações iniciais de cianeto de 10, 100, 500 e $1000 \mathrm{mg} / \mathrm{L}$, e proporção molar de $\left[\mathrm{H}_{2} \mathrm{O}_{2}+\mathrm{NaClO}\right]:\left[\mathrm{CN}^{-}\right]=2: 1$, foi possível atingir uma concentração final de cianeto menor do que $0,2 \mathrm{mg} / \mathrm{L}$, com $98,9 \%$ e $99 \%$ de remoção, a pH 11 e 9 , em apenas 2 e 20 minutos, respectivamente. Quando o cianeto foi oxidado por $\mathrm{H}_{2} \mathrm{O}_{2}$ e $\mathrm{NaClO}$ separadamente, para as mesmas condições experimentais, o peróxido de hidrogênio apenas oxidou o cianeto em $30 \%$ e $26 \%$, a pH 9 e 11, respectivamente, em 60 minutos de reação. Quando o cianeto foi oxidado com $\mathrm{NaClO}$, o cianeto atingiu uma concentração final menor do que $0,2 \mathrm{mg} / \mathrm{L}$, com uma remoção de $98 \%$ e 99\%, a pH 9 e 11, em 60 e 5 minutos de reação, respectivamente.

\section{Palavras-chave}

Oxigênio Singlete; oxidação do cianeto; peróxido de hidrogênio. 


\section{Abstract}

Arellano, Meryelem Tania Churampi; Luiz Alberto Cesar Teixeira (Advisor). Treatment of effluents containing cyanide by singlet oxygen generated by the reaction of hydrogen peroxide and sodium hypochlorite. Rio de Janeiro, 2012. 141 p. MSc. Dissertation Departamento de Engenharia de Materiais e Metalúrgica, Pontifícia Universidade Católica do Rio de Janeiro.

The synergistic combination of hydrogen peroxide and hypochlorite ion in water results in formation of the highly oxidizing intermediate species singlet oxygen $\left({ }^{1} \mathrm{O}_{2}\right)$, which is effective in the oxidation of free cyanide $\left(\mathrm{CN}^{-}\right)$in water. The process was fast and efficient over the studied $\mathrm{pH}$ range of 9-11, and up to an initial $\mathrm{CN}^{-}$concentration of $1000 \mathrm{mg} / \mathrm{L}$. For an initial $\left[\mathrm{CN}^{-}\right]=100 \mathrm{mg} / \mathrm{L}, \mathrm{pH}=9$, and molar ratio $\left(\left[\mathrm{H}_{2} \mathrm{O}_{2}\right]+[\mathrm{NaClO}]\right) /\left[\mathrm{CN}^{-}\right]=1: 1$ it was possible to achieve a final concentration of $\left[\mathrm{CN}^{-}\right]<0.2 \mathrm{mg} / \mathrm{L}\left(99.8 \%\right.$ reduction) in $\mathrm{t}=20 \mathrm{~min}$ at $25^{\circ} \mathrm{C}$ in a batch reaction. By comparison, the same reaction with either of the separate oxidants $\left(\mathrm{H}_{2} \mathrm{O}_{2}\right.$ or $\left.\mathrm{NaClO}\right)$ at the same molar ratio of oxidant $/ \mathrm{CN}^{-}=1: 1$ resulted in a maximum of $87 \%$ breakdown of the cyanide (using $\mathrm{NaClO}$ ) for the same $20 \mathrm{~min}$ reaction period.

\section{Keywords}

Singlet oxygen; oxidation cyanide; hydrogen peroxide. 


\section{Sumário}

1. Introdução 18

2. Objetivo 21

3. Revisão Bibliográfica 22

3.1. Principais Fontes de efluentes cianídricos 22

3.2. Aspectos físico-químicos do cianeto 23

3.2.1. Cianeto Livre 23

3.2.2. Compostos Simples de Cianeto 25

3.2.3. Complexos Ciano-Metálicos 25

3.3. Toxicidade do Cianeto 26

3.4. Limites de cianeto impostos pelos órgãos ambientais 28

3.5. Processos de Tratamento de Efluentes contendo cianeto 28

3.5.1. Degradação Natural 30

3.5.2. Processo com $\mathrm{H}_{2} \mathrm{O}_{2}$

3.5.3. Processo com $\mathrm{SO}_{2} / \mathrm{ar} \quad 34$

3.5.4. Cloração Alcalina $\quad 35$

3.5.5. Com Ácido de Caro $\left(\mathrm{H}_{2} \mathrm{SO}_{5}\right) \quad 38$

3.6. Processos Oxidativos Avançados (POA) 39

3.6.1 Fenton $\left(\left(\mathrm{H}_{2} \mathrm{O}_{2} / \mathrm{Fe}^{2+}\right) \quad 42\right.$

3.6.2 Sistema $\left(\mathrm{H}_{2} \mathrm{O}_{2} / \mathrm{UV}\right) \quad 44$

3.6.3 Sistema $\left(\mathrm{O}_{3} / \mathrm{UV}\right) \quad 46$

3.6.4 Processo $\mathrm{O}_{3} / \mathrm{H}_{2} \mathrm{O}_{2} / \mathrm{UV} \quad 48$

3.6.5 Oxigênio Singlete 49

3.6.5.1 Geração do Oxigênio Singlete 52

3.6.5.2 Reação de $\mathrm{H}_{2} \mathrm{O}_{2}$ e $\mathrm{NaClO} 54$

3.7. Comparação entre os métodos de tratamento de efluentes Cianetados 57

3.8. Cinética de oxidação $\quad 59$

4. Reagentes e Metodologia Analítica $\quad 64$

4.1. Reagente para os testes de oxidação 64

4.2. Características das Amostras 64

4.3. Planejamento Experimental 64

4.4. Procedimento Experimental 66 
4.4.1. Determinação de concentração do cianeto 67

4.4.2. Determinação da concentração do peróxido residual 68

4.4.3. Determinação da concentração do cloro livre 68

5. Apresentação e discussão dos resultados 69

5.1. Avaliação Físico-Química experimental 69

5.1.1. Influência do $\mathrm{pH}$ no processo de degradação do cianeto livre 69

5.1.1.1. Degradação com $\mathrm{H}_{2} \mathrm{O}_{2} \quad 69$

5.1.1.2. Degradação com $\mathrm{NaClO} \quad 72$

5.1.1.3. Degradação pela combinação do $\mathrm{H}_{2} \mathrm{O}_{2}$ e $\mathrm{NaClO} 76$

5.1.2. Influencia da Razão Molar na degradação do cianeto Livre 80

5.1.2.1. Influencia da razão molar do $\mathrm{H}_{2} \mathrm{O}_{2}: \mathrm{CN} \quad 80$

5.1.2.2. Influencia da razão molar do $\mathrm{NaClO}: \mathrm{CN} \quad 82$

5.1.2.3. Influencia da razão molar da combinação do $\left(\mathrm{H}_{2} \mathrm{O}_{2}+\right.$ $\begin{array}{ll}\mathrm{NaClO}): \mathrm{CN} & 88\end{array}$

5.1.3. Influência da concentração inicial do cianeto 96

5.1.3.1. Degradação com $\mathrm{H}_{2} \mathrm{O}_{2}$

5.1.3.2. Degradação com $\mathrm{NaClO} 98$

5.1.3.3. Degradação pela combinação do $\mathrm{H}_{2} \mathrm{O}_{2}$ e $\mathrm{NaClO} \quad 100$

5.2. Analise Comparativa entre a degradação do cianeto com $\mathrm{H}_{2} \mathrm{O}_{2}$ e $\mathrm{NaClO}$ aplicados separadamente e pelo sistema combinado $\left(\mathrm{H}_{2} \mathrm{O}_{2}+\mathrm{NaClO}\right) \quad 104$

5.3. Avaliação Cinética dos três processo estudados 110

5.3.1. Cinética da oxidação com peróxido de hidrogênio 110

5.3.2. Cinética de oxidação com hipoclorito de sódio 113

5.3.3. Cinética de oxidação pela combinação do peróxido de hidrogênio e hipoclorito de sódio. 117

5.3.3.1. Mecanismo simplificado do processo 117

5.4. Avaliação da velocidade de degradação inicial do cianeto 122

5.5. Análise da concentração do $\mathrm{H}_{2} \mathrm{O}_{2}$ residual 125

5.6. Análise da concentração do Cloro ativo residual 126

$\begin{array}{lll}\text { 6. Conclusões } & 128\end{array}$

7. Referências bibliográficas 129

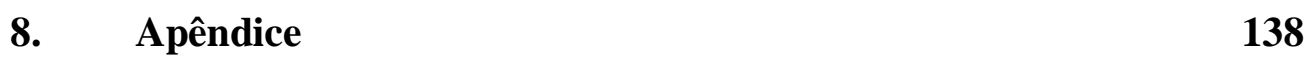




\section{Lista de Figuras}

Figura 1 Diagrama de predominância para o cianeto (Montalvo, 2004).

Figura 2 Principais processos de tratamento de efluente empregados na mineração de ouro.

Figura 3 Efeito do pH sobre a cinética de oxidação de cianeto livre ( Bonan, 1992).

Figura 4 Resumo das possíveis reações que podem ocorrer nos POA em presença de $\mathrm{O}_{3}, \mathrm{H}_{2} \mathrm{O}_{2}$ e radiação UV. Reações: adaptado de Gottschalk, 2000; Dezotti, 2008.

Figura 5 Esquema do sistema ULTROX $-\mathrm{O}_{3} / \mathrm{H}_{2} \mathrm{O}_{2} / \mathrm{UV}$ (EPA, 2008).

Figura 6 Curvas da energia potencial para os três estados excitados do oxigênio molecular (Maria de Rosa, Robert J., Crutchley, 2002).

Figura 7 Métodos para a geração do oxigênio singlete (krinsky, 1982).

Figura 8 Gráfico da primeira ordem de oxidação do cianeto livre $\left(1.1 \times 10^{-3} \mathrm{M}\right)$ por $(0,16 \mathrm{M})$ com $\mathrm{k}_{\mathrm{obs}}=2,93 \times 10^{-4} \mathrm{~s}^{-1}$ (LEAHY, 1990).

Figura 9, Reator montado para a oxidação do cianeto.

Figura 10 Remoção do cianeto com $\mathrm{H}_{2} \mathrm{O}_{2}$.Condições experimentais: pH 9 e 11, $\left[\mathrm{CN}^{-}\right]=10 \mathrm{mg} / \mathrm{L}$, razão molar $\left[\mathrm{H}_{2} \mathrm{O}_{2}\right]:\left[\mathrm{CN}^{-}\right]$ $=1: 1$ e $2: 1$.

Figura 11 Degradação do cianeto com $\mathrm{H}_{2} \mathrm{O}_{2}$. Condições experimentais: $\mathrm{pH}=9$ e $11 ;\left[\mathrm{CN}^{-}\right]=10 \mathrm{mg} / \mathrm{L} ;\left[\mathrm{H}_{2} \mathrm{O}_{2}\right]:\left[\mathrm{CN}^{-}\right]=1: 1$ e $2: 1$.

Figura 12 Velocidade inicial versus $\mathrm{pH}$ na degradação do cianeto com $\mathrm{H}_{2} \mathrm{O}_{2}$. Condições experimentais: $\mathrm{pH}=9$ e 11; $\left[\mathrm{CN}^{-}\right]=10 \mathrm{mg} / \mathrm{L}$; razão molar $\left[\mathrm{H}_{2} \mathrm{O}_{2}\right]:\left[\mathrm{CN}^{-}\right]=1: 1$ e $2: 1$.

Figura 13 Remoção do cianeto com $\mathrm{NaClO}$. Condições experimentais: pH 9 e 11; $\left[\mathrm{CN}^{-}\right]=10 \mathrm{mg} / \mathrm{L}$; razão molar $[\mathrm{NaClO}]$ : $\left[\mathrm{CN}^{-}\right]=1: 1$. 
Figura 14 Degradação do cianeto com hipoclorito de sódio.

Condições experimentais: pH 9 e $11 ;\left[\mathrm{CN}^{-}\right]=10 \mathrm{mg} / \mathrm{L}$;

razão molar $[\mathrm{NaClO}]:\left[\mathrm{CN}^{-}\right]=1: 1$ e $1: 2$.

Figura 15 Velocidade inicial versus $\mathrm{pH}$ na degradação do cianeto com $\mathrm{NaClO}$. Condições experimentais: pH 9 e 11; $\left[\mathrm{CN}^{-}\right]=10 \mathrm{mg} / \mathrm{L}$; e vários valores de razão molar $[\mathrm{NaClO}]:\left[\mathrm{CN}^{-}\right]$.

Figura 16 Remoção do cianeto com $\left(\mathrm{H}_{2} \mathrm{O}_{2}+\mathrm{NaClO}\right)$.

Condições experimentais: pH 9 e 11; $\left[\mathrm{CN}^{-}\right]=10 \mathrm{mg} / \mathrm{L}$; razão molar $\left[\mathrm{H}_{2} \mathrm{O}_{2}+\mathrm{NaClO}\right]:\left[\mathrm{CN}^{-}\right]=1: 1$.

Figura 17 Degradação do cianeto pela combinação de peróxido de hidrogênio e hipoclorito de sódio. Condições experimentais: pH 9 e 11; $\left[\mathrm{CN}^{-}\right]=10 \mathrm{mg} / \mathrm{L}$; razão molar $\left[\mathrm{H}_{2} \mathrm{O}_{2}+\mathrm{NaClO}\right]:\left[\mathrm{CN}^{-}\right]=1: 1$.

Figura 18 Velocidade inicial versus $\mathrm{pH}$ na degradação do cianeto com $\left(\mathrm{H}_{2} \mathrm{O}_{2}+\mathrm{NaClO}\right)$. Condições experimentais: $\mathrm{pH} 9$ e 11; $\left[\mathrm{CN}^{-}\right]=10 \mathrm{mg} / \mathrm{L}$; razão molar $\left[\mathrm{xH}_{2} \mathrm{O}_{2}+\mathrm{yNaClO}\right]:\left[\mathrm{CN}^{-}\right]$ $=(\mathrm{x}+\mathrm{y}): 1$ e $2: 1$.

Figura 19 Efeito da razão molar $\left[\mathrm{H}_{2} \mathrm{O}_{2}\right]:\left[\mathrm{CN}^{-}\right]$sobre a degradação do cianeto com $\mathrm{H}_{2} \mathrm{O}_{2}$. Condições experimentais: pH 9 e $11 ;\left[\mathrm{CN}^{-}\right]=10 \mathrm{mg} / \mathrm{L}$; razão molar $\left[\mathrm{H}_{2} \mathrm{O}_{2}\right]:\left[\mathrm{CN}^{-}\right]=1: 1$ e $2: 1$.

Figura 20 Velocidade inicial da degradação do cianeto com $\mathrm{H}_{2} \mathrm{O}_{2}$, Condições experimentais: $\mathrm{pH} 9$ e 11 ; $\left[\mathrm{CN}^{-}\right]=10 \mathrm{mg} / \mathrm{L}$; razão molar $\left[\mathrm{H}_{2} \mathrm{O}_{2}\right]:\left[\mathrm{CN}^{-}\right]=1: 1$ e $2: 1$.

Figura 21 Efeito da razão molar $[\mathrm{NaClO}]:\left[\mathrm{CN}^{-}\right]$sobre a degradação do cianeto com $\mathrm{NaClO}$. Condições experimentais: $\mathrm{pH}=9 ;\left[\mathrm{CN}^{-}\right]=10 \mathrm{mg} / \mathrm{L}$; e varias razões molares $[\mathrm{NaClO}]:\left[\mathrm{CN}^{-}\right]$.

Figura 22 Efeito da razão molar $[\mathrm{NaClO}]:\left[\mathrm{CN}^{-}\right]$sobre a degradação do cianeto com hipoclorito. Condições experimentais: $\mathrm{pH}=11 ;\left[\mathrm{CN}^{-}\right]=10 \mathrm{mg} / \mathrm{L}$; e deferentes razões molares $[\mathrm{NaClO}]:\left[\mathrm{CN}^{-}\right]$.

Figura 23 Efeito da razão molar $[\mathrm{NaClO}]:\left[\mathrm{CN}^{-}\right]$sobre a remoção do cianeto com hipoclorito. Condições experimentais: $\mathrm{pH}=11 ;\left[\mathrm{CN}^{-}\right]=10 \mathrm{mg} / \mathrm{L}$; e várias razões molares $[\mathrm{NaClO}]:\left[\mathrm{CN}^{-}\right]$. 
Figura 24 Efeito da razão molar $[\mathrm{NaClO}]:\left[\mathrm{CN}^{\top}\right]$ sobre a remoção do cianeto com hipoclorito. Condições experimentais: $\mathrm{pH}=9$; $\left[\mathrm{CN}^{-}\right]=10 \mathrm{mg} / \mathrm{L}$; e várias razões molares $[\mathrm{NaClO}]:\left[\mathrm{CN}^{-}\right]$.

Figura 25 Velocidade inicial da degradação do cianeto com NaClO. Condições experimentais: pH 9 e 11; [CN${ }^{-}=10$ mg/L; e varias razão molar $[\mathrm{NaClO}]:\left[\mathrm{CN}^{-}\right]$.

Figura 26 Efeito da razão molar $\left[\mathrm{H}_{2} \mathrm{O}_{2}+\mathrm{NaClO}\right]:\left[\mathrm{CN}^{-}\right]$sobre a degradação do cianeto com $\mathrm{H}_{2} \mathrm{O}_{2}+\mathrm{NaClO}$. Condições experimentais: $\mathrm{pH}=9 ;\left[\mathrm{CN}^{-}\right]=10 \mathrm{mg} / \mathrm{L}$; razão molar $\left[\mathrm{xH}_{2} \mathrm{O}_{2}+\right.$ $\mathrm{yNaClO}]:\left[\mathrm{CN}^{-}\right]=(\mathrm{x}+\mathrm{y}): 1=1: 1 \mathrm{e} 2: 1$.

Figura 27 Efeito da razão molar $\left[\mathrm{H}_{2} \mathrm{O}_{2}+\mathrm{NaClO}\right]:\left[\mathrm{CN}^{-}\right]$sobre a degradação do cianeto com $\mathrm{H}_{2} \mathrm{O}_{2}+\mathrm{NaClO}$ para gerar oxigênio singlete. Condições experimentais: $\mathrm{pH}=11$;

$\left[\mathrm{CN}^{-}\right]=10 \mathrm{mg} / \mathrm{L} ;$ razão molar $\left[\mathrm{xH}_{2} \mathrm{O}_{2}+\mathrm{yNaClO}\right]:\left[\mathrm{CN}^{-}\right]=$ $(\mathrm{x}+\mathrm{y}): 1=1: 1 \mathrm{e} 2: 1$.

Figura 28 Efeito da razão molar $\left[\mathrm{H}_{2} \mathrm{O}_{2}+\mathrm{NaClO}\right]:\left[\mathrm{CN}^{-}\right]$sobre a degradação do cianeto com $\mathrm{H}_{2} \mathrm{O}_{2}+\mathrm{NaClO}$ para gerar oxigênio singlete. Condições experimentais: $\mathrm{pH}=9$;

$\left[\mathrm{CN}^{-}\right]=10 \mathrm{mg} / \mathrm{L} ;$ razão molar $\left[\mathrm{xH}_{2} \mathrm{O}_{2}+\mathrm{yNaClO}\right]:\left[\mathrm{CN}^{-}\right]=$ $(\mathrm{x}+\mathrm{y}): 1=1: 1 \mathrm{e} 2: 1$.

Figura 29 Efeito da razão molar $\left[\mathrm{H}_{2} \mathrm{O}_{2}+\mathrm{NaClO}\right]:\left[\mathrm{CN}^{-}\right]$sobre a degradação do cianeto com $\mathrm{H}_{2} \mathrm{O}_{2}+\mathrm{NaClO}$ para gerar oxigênio singlete. Condições experimentais: $\mathrm{pH}=11$;

$\left[\mathrm{CN}^{-}\right]=10 \mathrm{mg} / \mathrm{L} ;$ razão molar $\left[\mathrm{xH}_{2} \mathrm{O}_{2}+\mathrm{yNaClO}\right]:\left[\mathrm{CN}^{-}\right]=$ $(\mathrm{x}+\mathrm{y}): 1=1: 1 \mathrm{e} 2: 1$.

Figura 30 Velocidade inicial da degradação do cianeto com $\mathrm{H}_{2} \mathrm{O}_{2}+\mathrm{NaClO}$. Condições experimentais: pH 9 e 11; $\left[\mathrm{CN}^{-}\right]=10 \mathrm{mg} / \mathrm{L}$; e varias razão molar $\left[\mathrm{xH}_{2} \mathrm{O}_{2}+\mathrm{yNaClO}\right]:\left[\mathrm{CN}^{-}\right]$ $=1: 1$.onde $\mathrm{x}+\mathrm{y}=1$.

Figura 31 Eficiência da remoção de cianeto pelo processo combinado $\mathrm{H} 2 \mathrm{O} 2$ e $\mathrm{NaClO}$ para gerar oxigênio singlete. Condições experimentais: pH 9 e 11, $\left[\mathrm{CN}^{-}\right]=10 \mathrm{mg} / \mathrm{L}$, $\left[\mathrm{xH}_{2} \mathrm{O}_{2}+\mathrm{yNaClO}\right]:\left[\mathrm{CN}^{-}=(\mathrm{x}+\mathrm{y}): 1=1: 1\right.$ e $2: 1$ e tempo $=2$ minutos. 
Figura 32 Efeito da c oncentração inicial do cianeto, sobre a degradação do cianeto com $\mathrm{H}_{2} \mathrm{O}_{2}$. Condições experimentais: pH 9 e 11; $\left[\mathrm{CN}^{-}\right]=10,100,500$ e 1000 mg/L; razão molar $\left[\mathrm{H}_{2} \mathrm{O}_{2}\right]:\left[\mathrm{CN}^{-}\right]=1: 1$.

Figura 33, Efeito da concentração inicial do cianeto, sobre a remoção do cianeto com $\mathrm{H}_{2} \mathrm{O}_{2}$. Condições experimentais: $\mathrm{pH} 9$ e $11 ;\left[\mathrm{CN}^{-}\right]=10,100,500$ e $1000 \mathrm{mg} / \mathrm{L}$; razão molar $\left[\mathrm{H}_{2} \mathrm{O}_{2}\right]:\left[\mathrm{CN}^{-}\right]=1: 1$.

Figura 34 Efeito da concentração inicial do cianeto, sobre a degradação do cianeto com $\mathrm{NaClO}$, Condições experimentais: $\mathrm{pH} 9$ e 11; $\left[\mathrm{CN}^{-}\right]=10,100,500$ e $1000 \mathrm{mg} / \mathrm{L}$; razão molar $[\mathrm{NaClO}]:\left[\mathrm{CN}^{-}\right]=1: 1$.

Figura 35 Efeito da concentração inicial do cianeto, sobre a remoção do cianeto com $\mathrm{NaClO}$. Condições experimentais: $\mathrm{pH}=11 ;\left[\mathrm{CN}^{-}\right]=10,100,500$ e $1000 \mathrm{mg} / \mathrm{L}$; razão molar $[\mathrm{NaClO}]:\left[\mathrm{CN}^{-}\right]=1: 1$.

Figura 36 Efeito da concentração inicial do cianeto, sobre a remoção do cianeto com $\mathrm{NaClO}$. Condições experimentais: $\mathrm{pH}=9 ;\left[\mathrm{CN}^{-}\right]=10,100,500$ e $1000 \mathrm{mg} / \mathrm{L}$; razão molar $[\mathrm{NaClO}]:\left[\mathrm{CN}^{-}\right]=1: 1$.

Figura 37 Efeito da concentração inicial do cianeto sobre a degradação do cianeto por combinação do $\mathrm{H}_{2} \mathrm{O}_{2} \mathrm{com}$ $\mathrm{NaClO}$, Condições experimentais: $\mathrm{pH}=11$; $\left[\mathrm{CN}^{-}\right]=10$, 100, 500 e $1000 \mathrm{mg} / \mathrm{L}$; razão molar $[\mathrm{NaClO}]:\left[\mathrm{CN}^{-}\right]=1: 1$.

Figura 38 Efeito da concentração inicial do cianeto sobre a remoção do cianeto por combinação do $\mathrm{H}_{2} \mathrm{O}_{2}$ com $\mathrm{NaClO}$, Condições experimentais: pH 9 e 11; [CN'] = 10 e 1000 mg/L; razão molar $[\mathrm{NaClO}]:\left[\mathrm{CN}^{-}\right]=1: 1$.

Figura 39 Comparação da degradação do cianeto por o $\mathrm{H}_{2} \mathrm{O}_{2}, \mathrm{NaClO}$ separados e combinados para gerar oxigênio singlete nas seguintes condições: Concentração inicial do cianeto $[\mathrm{CN}-]=100 \mathrm{mg} / \mathrm{L}, \mathrm{pH} 9$ e 11 , razão molar: $\left[\mathrm{H}_{2} \mathrm{O}_{2}\right]:\left[\mathrm{CN}^{-}\right]$ $=1: 1 ; \quad[\mathrm{NaClO}]:\left[\mathrm{CN}^{-}\right]=1: 1 ;\left[\mathrm{H}_{2} \mathrm{O}_{2}+\mathrm{NaClO}\right]:\left[\mathrm{CN}^{-}\right]=1: 1$. 
Figura 40 Comparação da degradação do cianeto por o $\mathrm{H}_{2} \mathrm{O}_{2}, \mathrm{NaClO}$ separados e combinados para gerar oxigênio singlete nas seguintes condições:

Concentração inicial do cianeto $\left[\mathrm{CN}^{-}\right]=100 \mathrm{mg} / \mathrm{L}, \mathrm{pH} 9$ e 11 , razão molares: $\left[\mathrm{H}_{2} \mathrm{O}_{2}\right]:\left[\mathrm{CN}^{-}\right]=2: 1 ; \quad[\mathrm{NaClO}]$

$:\left[\mathrm{CN}^{-}\right]=2: 1 ;\left[\mathrm{H}_{2} \mathrm{O}_{2}+\mathrm{NaClO}\right]:\left[\mathrm{CN}^{-}\right]=2: 1$.

Figura 41 Comparação da eficiência de degradação do $\mathrm{H}_{2} \mathrm{O}_{2}, \mathrm{NaClO}$ separados e combinados para gerar oxigênio singlete nas seguintes condições: concentração inicial do cianeto $\left[\mathrm{CN}^{-}\right]=100 \mathrm{mg} / \mathrm{L}, \mathrm{pH} 9$ e 11 , e razão molares: $\left[\mathrm{H}_{2} \mathrm{O}_{2}\right]:\left[\mathrm{CN}^{-}\right]=1: 1$ e $2: 1,[\mathrm{NaClO}]:\left[\mathrm{CN}^{-}\right]=1: 1$ e $2: 1$; $\left[\mathrm{H}_{2} \mathrm{O}_{2}+\mathrm{NaClO}\right]:\left[\mathrm{CN}^{-}\right]=1: 1$ e $2: 1$, tempo $=2$ minutos .

Figura 42 Comparação da velocidade inicial de degradação do cianeto pelos processos de $\mathrm{H}_{2} \mathrm{O}_{2}, \mathrm{NaClO}$ separados e combinados, nas seguintes condições: Concentração inicial do cianeto $\left[\mathrm{CN}^{-}\right]=100 \mathrm{mg} / \mathrm{L}, \mathrm{pH}=9$ e 11 , e razões molares $\left[\mathrm{H}_{2} \mathrm{O}_{2}\right]:\left[\mathrm{CN}^{-}\right]=2: 1,[\mathrm{NaClO}]:\left[\mathrm{CN}^{-}\right]=2: 1,\left[\mathrm{H}_{2} \mathrm{O}_{2}+\mathrm{NaClO}\right]:\left[\mathrm{CN}^{-}\right]=2: 1$.

Figura 43 Log da velocidade inicial da degradação do cianeto / log da concentração do peróxido de hidrogênio. Condições: concentração inicial do cianeto $\left[\mathrm{CN}^{-}\right]=10 \mathrm{mg} / \mathrm{L}, \mathrm{pH}=9$ e 11 , razão molares: $\left[\mathrm{H}_{2} \mathrm{O}_{2}\right]:\left[\mathrm{CN}^{-}\right]=1: 1$ e $2: 1$.

Figura 44 Log da velocidade inicial da degradação do cianeto / log da concentração do cianeto. Condições: concentração inicial do cianeto $\left[\mathrm{CN}^{-}\right]=10,100,500$ e $1000 \mathrm{mg} / \mathrm{L}, \mathrm{pH}=9$ e 11, razão molares: $\left[\mathrm{H}_{2} \mathrm{O}_{2}\right]:\left[\mathrm{CN}^{-}\right]=1: 1$.

Figura 45 Log da velocidade inicial da degradação do cianeto / log da concentração do hipoclorito de sódio. Condições: concentração inicial do cianeto $\left[\mathrm{CN}^{-}\right]=10 \mathrm{mg} / \mathrm{L}, \mathrm{pH}=9$ e 11 , razão molares: [NaClO]:[CN']=1:1 e 2:1.

Figura 46 Log da velocidade inicial da degradação do cianeto / log da concentração do cianeto . Condições: concentração inicial do cianeto $\left[\mathrm{CN}^{-}\right]=10,100,500$ e $1000 \mathrm{mg} / \mathrm{L}, \mathrm{pH}=9$ e 11, razão molares: [NaClO]:[ $\left.\mathrm{CN}^{-}\right]=1: 1$. 
Figura 47 Log da velocidade inicial da degradação do cianeto / log da concentração da combinação do peróxido de hidrogênio e hipoclorito de sódio. Condições: concentração inicial do cianeto $\left[\mathrm{CN}^{-}\right]=10 \mathrm{mg} / \mathrm{L}, \mathrm{pH}=9$ e 11 , razão molares: $\left[\mathrm{H}_{2} \mathrm{O}_{2}+\mathrm{NaClO}\right]:\left[\mathrm{CN}^{-}\right]=1: 1$ e $2: 1$.

Figura 48 Log da velocidade inicial da degradação do cianeto $/ \log$ da concentração do cianeto. Condições: concentração inicial do cianeto $\left[\mathrm{CN}^{-}\right]=10,100,500$ e $1000 \mathrm{mg} / \mathrm{L}, \mathrm{pH}=9$ e 11, razão molares: $\left[\mathrm{H}_{2} \mathrm{O}_{2}+\mathrm{NaClO}\right]:\left[\mathrm{CN}^{-}\right]=1: 1$.

Figura 49 Concentrações residuais do peróxido de hidrogênio. Condições experimentais: [CN-]=10mg/L, pH 9 e 11, e razão molares: $\left[\mathrm{H}_{2} \mathrm{O}_{2}\right]:[\mathrm{CN}-]=1: 1 ;\left[0,5 \mathrm{H}_{2} \mathrm{O}_{2}+0,5 \mathrm{NaClO}\right]:[\mathrm{CN}-]=1: 1$.

Figura 50 Concentrações residuais do $\mathrm{Cl}_{2}$. Condições experimentais: $[\mathrm{CN}-]=10 \mathrm{mg} / \mathrm{L}, \mathrm{pH} 9$ e 11, e razão molares: $\left[0,5 \mathrm{H}_{2} \mathrm{O}_{2}+0,5 \mathrm{NaClO}\right]:[\mathrm{CN}-]=1: 1$.

Figura A1-1 Degradação natural do cianeto. Condições experimentais: $[\mathrm{CN}-]=10 \mathrm{mg} / \mathrm{L}, \mathrm{pH} 9$ e 11 . 


\section{Lista de Tabelas}

Tabela 1 Processos industriais que geram efluentes contendo espécies cianídricas, Tendulkar \& Agnihotri, 2002 (Adaptado).

Tabela 2 Estabilidade relativa das espécies de Cianeto (Smith \& Mudder, 1991).

Tabela 3 Efeito da temperatura nas constantes das taxas de degradação de complexos metálicos de cianeto. (Smith \& Mudder, 1991).

Tabela 4 Resultados da oxidação do cianeto com peróxido de hidrogênio. Teixeira e Tavares, 1990 (Adaptado por Cinopoli, 2004).

Tabela 5 Potencial de oxirredução de vários oxidantes em água. (Handbook, 1985, Dezotti, 2008).

Tabela 6 Reações e os potenciais padrões para oxidantes comunes (Parga \& Cocke, 2001).

Tabela 7 Processos de Oxidação Avançados homogêneos e heterogêneos. (Livro de Processos e técnicas para controle ambiental de efluentes líquidos, Márcia Dezotti, 2008).

Tabela 8 Mecanismos de reações do processo $\mathrm{H}_{2} \mathrm{O}_{2} / \mathrm{UV}$ (Beltrán, 2004 e Kurniawan, 2006).

Tabela 9 Os estados eletrônicos de oxigênio molecular e suas propriedades. Bellus, 1979. (Adaptado).

Tabela 10 Transições de moléculas simples e transições de moléculas simultâneas. Kearns, 1971.

Tabela 11 Correlação do $\tau$ do ${ }^{1} \mathrm{O}_{2}$ com a absorbância de cada solvente a $1270 \mathrm{~nm}$. Ronsein, 2008.

Tabela 12 Comparação entre os processos mais usados para tratamento de efluentes contendo espécies cianídricas (Sarla. M., et al., 2004, Adaptado).

Tabela 13 Constante de velocidade da oxidação do cianeto por processos químicos e fotoquímicos. (Sarla M., et al., 2004). 
Tabela 14 Variáveis estabelecidas no planejamento experimental, nos seus níveis inferior e superior respectivamente.

Tabela 15 Valores calculados de ordem de reação e constante de velocidade aparente, na Figura 43.

Tabela 16 Valores calculados de ordem de reação e constante de velocidade aparente, na Figura 44.

Tabela 17 Valores calculados de ordem de reação e constante de velocidade aparente, na Figura 45.

Tabela 18 Valores calculados de ordem de reação e constante de velocidade aparente, na Figura 46.

Tabela 19 Valores calculados de ordem de reação e constante de velocidade aparente, na Figura 47.

Tabela 20 Valores calculados de ordem de reação e constante de velocidade aparente, na Figura 48.

Tabela 21Velocidades Iniciais do cianeto oxidado por $\mathrm{H}_{2} \mathrm{O}_{2}$, $\mathrm{NaClO}$ separadamente.

Tabela 22 Velocidades iniciais do cianeto oxidado pela combinação $\mathrm{H}_{2} \mathrm{O}_{2}+\mathrm{NaClO}$ (Oxigênio Singlete). Condicções experimentais: pH 9 e 11, $\left[\mathrm{CN}^{-}\right]=10 \mathrm{mmg} / \mathrm{L}$, razão molar $\left[\mathrm{CN}^{-}\right]:\left[\mathrm{x} \mathrm{H}_{2} \mathrm{O}_{2}+\mathrm{y} \mathrm{NaClO}\right]=1:(\mathrm{x}+\mathrm{y})$, onde $\mathrm{x}$ e $\mathrm{y}$ adotam deferentes valores de razão molar.

Tabela A1-1 Velocidades iniciais do cianeto oxidado por atuação isolada de $\mathrm{H}_{2} \mathrm{O}_{2}, \mathrm{NaClO}$ e pela combinação de $\mathrm{H}_{2} \mathrm{O}_{2}+\mathrm{NaClO}$ (Oxigênio Singlete) condições experimentais: $\mathrm{pH}=9$ e $11,\left[\mathrm{CN}^{-}\right]=100,500$ e $1000 \mathrm{mg} / \mathrm{L}$, razão molar de $\left[\mathrm{CN}^{-}\right]:\left[\mathrm{H}_{2} \mathrm{O}_{2}\right] ;\left[\mathrm{CN}^{-}\right]:[\mathrm{NaClO}] ;\left[\mathrm{CN}^{-}\right]:\left[\mathrm{H}_{2} \mathrm{O}_{2}+\mathrm{NaClO}\right]=1: 1 \mathrm{e}$ $2: 1$.

Tabela A1-2 Peróxido residual e $\mathrm{Cl}_{2}$ residual após a degradação do cianeto, condições experimentais: pH 9 e 11, $[\mathrm{CN}-]=10 \mathrm{mg} / \mathrm{L}$, razão molar de 1:1 (diferentes dosagens de oxidantes) e $2: 1$.

Tabela A1-3 Concentrações da oxidação do cianeto por degradação natural. Condições experimentais: pH 9 e 11, $\left[\mathrm{CN}^{-}\right]_{0}=10 \mathrm{mg} / \mathrm{L}, \mathrm{T}=25 \pm 1^{\circ} \mathrm{C}$. 